\title{
Uso de Plantas com Finalidade Medicinal por Pessoas Vivendo com HIV/ AIDS em Terapia Antirretroviral
}

\section{Use of plants With Medicinal Purposes by People Living with HIV/ AIDS on Antiretroviral Therapy}

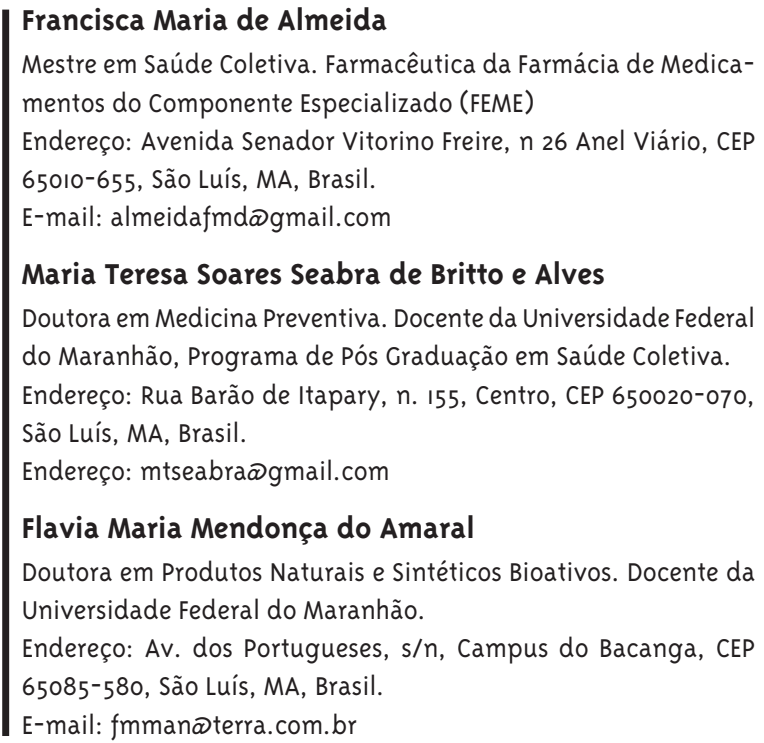

Flavia Maria Mendonça do Amaral

Doutora em Produtos Naturais e Sintéticos Bioativos. Docente da Universidade Federal do Maranhão.

Endereço: Av. dos Portugueses, s/n, Campus do Bacanga, CEP 65085-580, São Luís, MA, Brasil.

E-mail: fmman®terra.com.br

\section{Resumo}

Este foi um estudo observacional, transversal analítico realizado em ambulatório de referência do Estado do Maranhão-Brasil, no período de maio de 2009 a fevereiro de 2010, com o objetivo de estudar o uso de plantas com finalidade medicinal entre pessoas vivendo com HIV/AIDS, em uso de antirretrovirais. Um total de 339 pessoas respondeu um questionário abordando o uso de plantas e características demográficas, socioeconômicas, comportamentais, relacionadas à soropositividade e ao uso de antirretrovirais. A prevalência de utilização de plantas foi de $34,81 \%$. As mais utilizadas foram: Turnera ulmifolia (12,09\%); Melissa officinalis (10,62\%); Plectranthus barbatus (7,67\%); Cymbopogan citratus (capim limão) (4,72\%) e Mentha spp. (hortelã) (2,36\%). A maioria das pessoas (96,61\%) referiu melhora após a utilização. Um percentual de 75,42\% dos usuários de plantas não informou essa prática ao médico. Entre os que informaram o uso, 55,17\% afirmaram que o médico estava de acordo e somente uma pessoa foi orientada a interromper o uso $(3,45 \%)$. Apenas um médico (3,45\%) indicou o uso de plantas. A análise ajustada evidenciou diferença para uso de plantas em relação ao sexo feminino $(\mathrm{RP}=1,58,95 \%$ IC 1,15-2,15 p o,004) e à orientação sexual do tipo homossexual ( $\mathrm{RP}=0,63$ IC o,44-0,90 p o,012). Este estudo aponta para a necessidade de melhor diálogo entre médico e pacientes sobre o uso de plantas com finalidade medicinal, alertando sobre possíveis perigos quando associados aos antirretrovirais, especialmente entre usuários do sexo feminino ou com prática do tipo homossexual.

Palavras-chave: Plantas com finalidade medicinal; HIV; Antirretrovirais. 


\section{Abstract}

It is an observational, analytic study, developed at a hospital in Maranhao-Brazil, from May-2009 to February-2010. The objective was to study the use of plants with medicinal purpose in people living with HIV/AIDS and using retroviral therapy. A total of 339 (three hundred and thirty-nine) people answered a questionnaire about the use of plants and demographic, socioeconomic, behavioral characteristics, including those related to HIV status and use of antiretroviral therapy The prevalence of the use of plants with medicinal purpose was $34,81 \%$. The most often used were: Turnera ulmifolia (chanana) (12,09\%), Melissa officinalis (erva cidreira,) (10,62\%), Plectranthus barbatus (boldo) (7,67\%), Cymbopogan citratus (capim limão) (4,72\%) and Mentha spp. (hortelã) (2,36\%). Most people interviewed (96,61\%) reported improvement after use. A rate of $75,42 \%$ of the plant users had not reported their practice to a medical doctor. Among respondents who reported use, $55.17 \%$ said their doctor agreed to it, and only one person was advised to discontinue the use $(3,45 \%)$; only one doctor $(3,45 \%)$ indicated the use of plants. Multivariate analysis showed differences for the use of plants in relation to gender (female PR=1,58, 95\% CI 1,15-2,15 p o,o 04) and homosexual practices (PR= 0,63, CI o,44- 0,90 p o,012). This study highlights the need for a better dialogue between doctors and patients about the use of plants with medicinal purposes, and warns about possible dangers when they are combined with antirretroviral therapy, particularly between female and homossexual users.

Keywords: Plants With Medicinal Purpose; HIV; Antiretrovirals.

\section{Introdução}

A utilização de plantas com finalidade terapêutica é uma prática comum no mundo e tem crescido nos últimos anos (Lee e col., 2006). Há evidências de que a utilização seja maior entre indivíduos portadores de doenças crônicas, sobretudo vivendo com HIV/ AIDS em terapia antirretroviral (Hsiao e col, 2003). Estudos têm demonstrado que muitos destes indivíduos não informam aos seus cuidadores a respeito desta prática (Peltzer e col., 2008; Hsiao e col., 2003), o que pode comprometer a eficácia dos antirretrovirais como resultado de interações medicamentosas ou potencialização de efeitos adversos, incluindo toxicidade (Littlewood e Vanable, 2008).

Não há evidências que apoiem a eficácia e segurança de terapia complementar e/ou alternativa no tratamento para HIV/AIDS. Em contrapartida, os medicamentos antirretrovirais têm impacto na qualidade de vida e na redução da mortalidade dos indivíduos (Liu e col., 2005).

Os motivos que levam estes pacientes a utilizarem plantas como recursos terapêuticos são, sobretudo, a necessidade de: combater reações adversas causadas pelos medicamentos antirretrovirais (Chinsembu, 2009); aumentar imunidade; combater vírus; diminuir stress, evitar perda de peso, combater diarréia e melhorar a qualidade de vida (Chang e col., 2003; Passos e col., 2009).

Entre as plantas utilizadas para estes fins estão: Plectranthus barbatus (boldo); Matricaria chamomilla (camomila); Melissa officinalis (erva cidreira); Cymbopogon citratus (capim santo) (Passos e col., 2009); Hypericum perforatum (erva de São João) e Allium sativum (alho) (Lee e col., 2006). Entretanto, algumas destas plantas já foram descritas na literatura como potenciais causadores de interações medicamentosas, a exemplo de Hypericum perforatum, que contém substâncias como hiperforin, capazes de induzir as enzimas do citocromo $\mathrm{P} 45^{\circ}$ e reduzir a concentração dos agentes inibidores de transcriptase reversa, análogos de nucleosídeo e inibidores de protease (Lee e col., 2006).

Sabe-se que o Brasil tem estrutura para oferecer assistência à saúde por meio de plantas como recurso terapêutico, fundamentado nos resultados das pesquisas da flora, com garantia de emprego 
seguro (Eldin e Dunford, 2001; Yunes e col., 2001). Nesse sentido, em 2006, foram publicadas a Política Nacional de Plantas Medicinais e Fitoterápicos (Brasil, 2006) e a Política Nacional de Práticas Integrativas e Complementares no Sistema Único de Saúde (SUS) (MS, 2006), apresentando como diretrizes: o provimento do acesso a plantas medicinais e fitoterápicos; o incentivo à pesquisa e desenvolvimento de medicamentos a partir de plantas, priorizando a biodiversidade do país.

Dutra (2009), porém, alerta que as pesquisas visando à validação de plantas são insuficientes e que muitas plantas continuam a ser utilizadas com base apenas no conhecimento do uso popular. Esse saber popular do uso de plantas é importante na descoberta de espécies vegetais potenciais; todavia, o uso de plantas com fins terapêuticos, sem estudos de validação, constitui risco de toxicidade, considerando que espécies vegetais podem interferir na terapêutica de referência. (Amaral, 2007; Amaral e col., 2001; Matos, 2000).

Assim, este estudo teve como objetivo estimar a prevalência de uso de plantas com finalidade medicinal por pessoas vivendo com HIV/AIDS em um ambulatório de referência do Estado do MaranhãoBrasil e, ainda, identificar as plantas utilizadas, suas características e os fatores associados ao seu uso.

\section{Material e Métodos}

Trata-se de um estudo observacional, transversal analítico. A população de estudo foi composta por pessoas vivendo com HIV/AIDS, maiores de 18 (dezoito) anos, cadastradas no ambulatório de referência do Hospital Presidente Getúlio Vargas (HPGV), em São Luís - Maranhão, Brasil, no período de maio de 2009 a fevereiro de 2010.

Todo paciente em regime ambulatorial, em uso regular de antirretrovirais há pelo menos o1 (um) mês foi convidado a participar da pesquisa.

Considerando-se a escassez de pesquisas nacionais que estimassem o uso de plantas com finalidade medicinal em pessoas em uso de terapia antiretroviral, optou-se por realizar um estudo piloto nas duas primeiras semanas da pesquisa. A prevalência obtida neste estudo piloto foi 30\%.

Respaldando-se nesta prevalência de 30\% e sabendo-se que a população cadastrada para o recebimento de antirretrovirais era de 1.000 (mil) pacientes, consideramos um erro de $4 \%$ e intervalo de confiança de $95 \%$ : assim estabeleceu-se o tamanho da amostra necessária para o estudo, de 335 (trezentos e trinta e cinco) pacientes.

O estudo foi submetido ao Comitê de Ética em Pesquisa do Hospital Universitário Presidente Dutra e aprovado (Parecer $n^{\circ}$ 187/2009). Todos os participantes foram solicitados a assinar o Termo de Consentimento Livre e Esclarecido (TCLE) antes da coleta de dados,.

Para a coleta de dados, foi utilizado questionário semiestruturado, com questões sobre o uso de plantas e informações demográficas, socioeconômicas, comportamentais, referentes à soropositividade e ao uso de antirretrovirais. Dados de quantificação de carga viral do HIV e contagem de linfócitos T CD4 foram coletados do prontuário médico. Foi utilizada ficha para solicitação de medicamentos antirretrovirais da farmácia do ambulatório, para verificar o esquema terapêutico empregado.

O uso de plantas foi considerado variável dependente. Os pacientes que fizeram uso de plantas responderam questões adicionais: como se sentiu após o uso; se o médico havia sido comunicado sobre esta utilização; se o médico havia concordado com o uso; se foi orientado pelo médico a interromper e quem indicou a utilização de plantas.

Para identificação dos gêneros e espécies vegetais utilizadas, foi considerada similaridade de gêneros e espécies da região, registrados em estudos etnobotânicos desenvolvidos anteriormente (Rêgo, 1995; Coutinho e col., 2002; Amaral, 2007; Monteles e Pinheiro, 2007).

As variáveis independentes utilizadas foram: demográficas (idade, sexo, cor de pele, estado marital, residência e procedência); socioeconômicas (escolaridade, trabalho e renda); comportamentais (orientação sexual e religião) e soropositividade (tempo de HIV, categoria de transmissão, número de linfócito TCD4 e carga viral). Em relação ao uso de antirretrovirais, a variável utilizada foi o uso de inibidores de protease. Todas as variáveis foram dicotomizadas para melhor efeito da análise estatística.

Para análise dos dados foi empregado o Programa Stata 10.o. Inicialmente foi realizada a análise descritiva das variáveis, seguida do cálculo de pre- 
valência do uso de plantas por três ou mais pessoas e seus respectivos intervalos de confiança em nível de $95 \%$. A análise não ajustada foi realizada observando se as razões de prevalência e os respectivos intervalos de confiança em $95 \%$, estimando diferenças nas proporções por meio do teste qui-quadrado.

A análise multivariada foi realizada por meio da Regressão de Poisson, que apresenta como medida de efeito a razão de prevalência. Isto permite interpretações mais precisas, uma vez que a razão de chances superestima a magnitude de eventos com prevalência superior a 10\% (Barros e Hirakata, 2003).

As variáveis com $p$-valor menor ou igual a 0,20 foram incluídas no modelo de Poisson multivariável robusto. Para seleção das variáveis significativas, foi empregado o método stepwise. Também foram calculados a razão de prevalência e seu respectivo intervalo de confiança, com nível de significância de 5\%.

\section{Resultados}

Dos 349 pacientes que concordaram em participar do estudo, 339 foram incluídos na análise e 10 pacientes foram excluídos por insuficiência de dados.

A prevalência de uso de plantas pela população em estudo foi de 34,81\% (n 118 IC 95\% 29,0-40,o). Foi relatada a utilização de 56 espécies vegetais distintas. As mais utilizadas foram: Turnera ulmifolia (chanana), com prevalência de 12,09\% (n 41 IC 95\% 8,0-16,0), seguida por Melissa officinalis (erva cidreira) com prevalência de 10,62\% ( n 36 IC 95\% 7,0-14,o); Plectranthus barbatus (boldo) 7,67\% (n 26 IC 95\%5,o-11,o); Cymbopogan citratus (capim santo) 4,72\% (n 16 IC 95\% 2,o-7,o); Mentha spp. 2,36\% (hortelã) (n 8 IC 95\% 1,o-4,o); Matricaria chamomilla (camomila) 2,06\% (n 7 IC 95\% o,8-4,o); Litharae brasiliensis (aroeira) 1,77\% (n 6 IC 95\% o,63,o); Aloe vera (babosa) 1,47\% (n 5 IC 95\% o,4-3,o); Arrabidaea chica (pariri) 1,47\% (n 5 IC 95\% 0,4-3,o); Phyllanthus niruri (quebra-pedra) 1,18\% (n 4 IC 95\% 0,3-2,0); Maytenus ilicifolia (espinheira santa) 1,18\% (n 4 IC 95\% o,3-2,o) e Bixa orellana (urucum) o, 89\% (n 3 IC $95 \%$ o,1-2,o).

Das pessoas que estavam utilizando plantas, 96,61\% (114) referiram sentir-se melhor após utilizálas, mas 75,42\% (89) desses entrevistados não informaram ao médico assistente sobre tal utilização. Dos 29 (vinte e nove) indivíduos que informaram ao médico sobre a utilização de plantas, 55,17\% (16) disseram que ele concordava com a utilização; enquanto 37,93\% (11) relataram que o médico, ao ser informado sobre o uso de plantas, não manifestou concordância ou discordância a cerca de tal prática (Tabela 1).

Tabela I - Uso de plantas por pessoas vivendo com HIV/ AIDS em terapia antirretroviral, em ambulatório de referência do Maranhão, 2010

\begin{tabular}{|c|c|c|c|}
\hline Variáveis & Modalidades & $n$ & $\%$ \\
\hline \multirow[t]{3}{*}{ Situação após uso } & Pior & 1 & 0,86 \\
\hline & Melhor & 114 & 96,61 \\
\hline & Nem melhor nem pior & 3 & 2,54 \\
\hline \multirow[t]{2}{*}{ Informação sobre uso ao médico } & Sim & 29 & 24,58 \\
\hline & Não & 89 & 75,42 \\
\hline \multirow[t]{3}{*}{ Concordância do médico com o uso } & Sim & 16 & 55,17 \\
\hline & Não & 2 & 6,90 \\
\hline & Nem sim/ nem não & 11 & 37,93 \\
\hline \multirow[t]{2}{*}{ Orientação sobre interrupção do uso } & Sim & 1 & 3,45 \\
\hline & Não & 28 & 96,55 \\
\hline \multirow[t]{5}{*}{ Indicação do uso de plantas } & Médico & 1 & 3,45 \\
\hline & Família, amigos ou por indicação própria & 17 & 58,62 \\
\hline & Jornal, revista, livros ou internet & 6 & 20,69 \\
\hline & Outros profissionais de saúde & 4 & 13,79 \\
\hline & Outros & 1 & 3,45 \\
\hline
\end{tabular}


Tabela 2 - Análise de Poisson não ajustada do uso de plantas por pessoas vivendo com HIV/AIDS em terapia antirretroviral em ambulatório de referência do Maranhão, 2010

\begin{tabular}{|c|c|c|c|c|c|c|c|}
\hline Caracteristicas & USO & Não & $\%$ & Sim & $\%$ & $\mathrm{RP}(95 \% \mathrm{Cl})$ & $p$ \\
\hline \multicolumn{8}{|l|}{ Demográficas } \\
\hline \multirow[t]{2}{*}{ Faixa etária } & 18 a 39 anos & 123 & 55,66 & 57 & 48,3 & & \\
\hline & 40 anos ou mais & 98 & 44,34 & 61 & 51,7 & $1,21(0,90-1,62)$ & 0,19 \\
\hline \multirow[t]{2}{*}{ Sexo } & Masculino & 138 & 62,44 & 62 & 52,5 & & \\
\hline & Feminino & 83 & 37,56 & 56 & 47,5 & $1,29(0,97-1,73)$ & 0,07 \\
\hline \multirow[t]{2}{*}{ Cor de pele } & Branca & 32 & 14,48 & 22 & 18,6 & & \\
\hline & Não branca & 189 & 85,52 & 96 & 81,4 & $0,82(0,57-1,18)$ & 0,3 \\
\hline \multirow[t]{2}{*}{ Estado Marital } & Sem companheiro & 149 & 67,42 & 86 & 72,9 & & \\
\hline & Com companheiro & 72 & 32,58 & 32 & 27,1 & $0,84(0,61-1,17)$ & 0,3 \\
\hline \multirow[t]{2}{*}{ Residência } & Rural & 32 & 14,55 & 17 & 14,4 & & \\
\hline & Urbana & 188 & 85,45 & 101 & 85,5 & $1,00(0,66-1,52)$ & 0.97 \\
\hline \multirow[t]{2}{*}{ Procedência } & Interior do estado & 65 & 29,55 & 46 & 39 & & \\
\hline & Região metropolitana & 155 & 70,45 & 72 & 61 & $0,76(0,57-1,02)$ & 0.07 \\
\hline \multicolumn{8}{|l|}{ Socioeconômicas } \\
\hline \multirow[t]{2}{*}{ Escolaridade } & Até ensino médio & 206 & 93,21 & 108 & 91,5 & & \\
\hline & Mais que ensino médio & 15 & 6,79 & 10 & 8,47 & $1,16(0,70-1,92)$ & 0,55 \\
\hline \multirow[t]{2}{*}{ Trabalho } & Não está trabalhando & 84 & 38,01 & 50 & 42,4 & & \\
\hline & Está trabalhando & 137 & 61,99 & 68 & 57,6 & $0,88(0,66-1,19)$ & 0,43 \\
\hline \multirow[t]{2}{*}{ Renda/salário mínimo } & Até 2 salários & 207 & 93,67 & 107 & 90,7 & & \\
\hline & Mais de 2 salários & 14 & 6,33 & "1 & 9,32 & $1,29(0,80-2,06)$ & 0,28 \\
\hline \multicolumn{8}{|l|}{ Comportamentais } \\
\hline \multirow[t]{2}{*}{ Orientação sexual } & Relação homo ou bissexual & 39 & 17,65 & 28 & 23,7 & & \\
\hline & Relação heterossexual & 182 & 82,35 & 90 & 76,3 & $0,79(0,56-1,10)$ & 0,16 \\
\hline \multirow[t]{2}{*}{ Religião } & Não católica & 85 & 38,46 & 44 & 37,3 & & \\
\hline & Católica & 136 & 61,54 & 74 & 62,7 & $1,03(0,76-1,39)$ & 0,83 \\
\hline \multicolumn{8}{|l|}{ Soropositividade } \\
\hline \multirow[t]{2}{*}{ Tempo de HIV } & Menos de dois anos & 43 & 19,46 & 30 & 25,4 & & \\
\hline & Dois ou mais anos & 178 & 80,54 & 88 & 74,6 & $0,80(0,58-1,11)$ & 0,18 \\
\hline \multirow[t]{2}{*}{ Categ. de transm.o } & Transmissão sexual & 149 & 67,42 & 77 & 65,3 & & \\
\hline & Outras & 72 & 32,58 & 41 & 34,8 & $1,06(0,78-1,44)$ & 0,68 \\
\hline \multirow[t]{2}{*}{ Nde Linfócitos T CD4/cel/ml } & $\mathrm{TCD}_{4}$ menor que 200 & 58 & 26,73 & 27 & 23,3 & & \\
\hline & $\mathrm{TCD}_{4}$ maior ou igual a 200 & 159 & 73,27 & 89 & 76,7 & $1,12(0,79-1,60)$ & 0,49 \\
\hline \multirow[t]{2}{*}{ Carga Viral } & Indetectável & 137 & 63,43 & 75 & 65,8 & & \\
\hline & Detectável & 79 & 36,57 & 39 & 34,2 & $0,93(0,68-1,28)$ & 0,67 \\
\hline \multicolumn{8}{|l|}{ Antirretrovirais } \\
\hline & Não usa inibidores de protease & 129 & 58,9 & 70 & 59,3 & & \\
\hline & Usa inibidores de protease & 90 & 41,1 & 48 & 40,7 & $0,98\left(0,73^{-1,33)}\right.$ & \\
\hline
\end{tabular}


Neste estudo, foi constatado que somente um indivíduo foi orientado a interromper a utilização de plantas com finalidade medicinal (3,45\%). A maioria dos entrevistados $(58,62 \%)$ declarou indicação para uso de plantas principalmente de amigos, da família ou por conta própria. Apenas um entrevistado referiu essa indicação feita pelo médico $(3,45 \%)$ (Tabela 1).
Na análise não ajustada, nenhuma das variáveis estudadas apresentou diferença estatisticamente significante (p o,05) para uso de plantas (Tabela 2). A análise ajustada evidenciou significância estatística para uso de plantas por mulheres (RP 1,58; IC 1,15-2,15; p o,004), assim como para pessoas com orientação homossexual ( $\mathrm{RP}=0,63$; IC o,44-o,9o; $p$ o,o12) (Tabela 3).

Tabela 3 - Análise de Poisson ajustada do uso de plantas por pessoas vivendo com HIV/AIDS em terapia antirretroviral em ambulatório de referência do Maranhão, 2010.

\begin{tabular}{|c|c|c|c|c|c|c|c|}
\hline Caracteristicas & Uso & Não & $\%$ & Sim & $\%$ & $\mathrm{RP}(95 \% \mathrm{Cl})$ & $\mathrm{p}$ \\
\hline \multicolumn{8}{|l|}{ Demográficas } \\
\hline \multirow[t]{2}{*}{ Faixa Etária } & 18 a 39 anos & 123 & 55,66 & 57 & 48,31 & & \\
\hline & 40 anos ou mais & 98 & 44,34 & 61 & 51,69 & $1,34(1,00-1,80)$ & 0,04 \\
\hline \multirow[t]{2}{*}{ Sexo } & Masculino & 138 & 62,44 & 62 & 52,54 & & \\
\hline & Feminino & 83 & 37,56 & 56 & 47,46 & $1,58(1,15-2,15)$ & 0,004 \\
\hline \multirow[t]{2}{*}{ Procedência } & Interior do estado & 65 & 29,55 & 46 & 38,98 & & \\
\hline & Região metropolitana & 155 & 70,45 & 72 & 61,02 & $0,78(0,58-1,04)$ & 0,09 \\
\hline \multicolumn{8}{|l|}{ Comportamentais } \\
\hline \multirow[t]{2}{*}{ Orientação sexual } & Homo ou bissexual & 39 & 17,65 & 28 & 23,73 & & \\
\hline & Relação heterossexual & 182 & 82,35 & 90 & 76,27 & $0,63(0,44-0,90)$ & 0,01 \\
\hline \multicolumn{8}{|l|}{ Soropositividade } \\
\hline \multirow[t]{2}{*}{ Tempo de HIV } & Menos de dois anos & 43 & 19,46 & 30 & 25,42 & & \\
\hline & Dois ou mais anos & 178 & 80,54 & 88 & 74,58 & $0,74(0,54-1,04)$ & 0,09 \\
\hline
\end{tabular}

\section{Discussão}

A prevalência do uso de plantas com finalidade medicinal, constatada neste estudo (34,81\%) confirmou a hipótese de taxa de uso na população estudada superior a 30\%. Estudo semelhante realizado em ambulatório de Brasília evidenciou prevalência de $52,3 \%$ (Sales e col., 2008); outro estudo desenvolvido em Santa Catarina indicou prevalência de 60\% (Passos e col., 2009).

Considerando a influência indígena, africana e européia, bem como os costumes relacionados ao uso tradicional de plantas como recurso terapêutico (Macedo e col., 2007; Monteles e Pinheiro, 2007), além da biodiversidade e das condições socioeconômicas do Maranhão (Moura, 2004; Feitosa e Trovão, 2006), esperava-se uma prevalência do uso de plantas na amostra em estudo mais elevada.
Essa taxa de prevalência relativamente baixa do uso de plantas por pessoas com HIV/AIDS desperta atenção também quando comparada a estudos etnofarmacológicos realizados na região. (Coutinho e col., 2002; Amaral, 2007; Monteles e Pinheiro 2007). Esses estudos indicam prevalência superior a $50 \%$ no uso de espécies vegetais para fins terapêuticos em outros agravos à saúde. Dentre os motivos para este uso é referido o alto custo dos medicamentos sintéticos e dificuldade de acesso como justificativa para o emprego de plantas.

A eficácia da Assistência Farmacêutica do Maranhão na cobertura terapêutica dos antirretrovirais pode justificar a relativa baixa prevalência do uso de plantas na amostra em estudo, visto que pessoas vivendo com HIV/AIDS têm tido acesso ao tratamento de referência, o que pode influenciar a prática de uso de terapias complementares/alternativas. 
Convém enfatizar que o uso de plantas com finalidade medicinal em HIV/AIDS pode sofrer a influencia, no Brasil, da grande abrangência do Programa de Assistência à Saúde para pessoas vivendo com HIV/AIDS e da distribuição universal e gratuita dos medicamentos antirretrovirais (Meiners e col., 2008; Paula e col., 2009).

Os antirretrovirais têm eficácia terapêutica comprovada, diferentemente de plantas usadas com finalidade medicinal, cujos estudos de validação e comprovação terapêutica segura para emprego no tratamento de HIV/AIDS ainda são inexistentes (Liu e col., 2005).

As espécies vegetais mais utilizadas, identificadas neste estudo, foram: Turnera ulmifolia (chanana); Melissa officinalis (erva cidreira); Plectranthus barbatus (boldo) e Cymbopogan citratus (capim limão). Tais resultados concordam parcialmente com a pesquisa desenvolvida por Passos e colaboradores (2009), em Santa Catarina-Brasil, identificando Melissa officinalis (erva cidreira) (57\%), Mentha piperita (hortelã) (33,9\%) e Peumus boldus (boldo) entre as plantas mais utilizadas por pessoas vivendo com HIV/AIDS.

O Hipericum perforatum (erva de São João) e Allium sativum (alho), também têm sido frequentemente utilizadas por pessoas com sorologia positiva para HIV (Lee e col., 2006).

Turnera ulmifolia, espécie conhecida popularmente na região como chanana, foi identificada como planta com maior freqüência de citação neste estudo (12,09\%). Algumas espécies do gênero Turnera são utilizadas como expectorantes e afrodisíacos (Rêgo, 1995; Barbosa e col., 2007).

Estudos de atividade biológica indicaram atividade de Turnera ulmifolia no tratamento de afecções respiratórias e como anti-inflamatório (Antonio e Souza 1998; Arantes, 2005; Nascimento e col., 2006). Estudos de investigação química desenvolvidos com espécies do gênero Turnera, identificaram presença de flavonóides (Piacente e col., 2002), metabólito secundário capaz de inibir as enzimas do citocromo P 450, o que interfere na terapêutica com antirretrovirais (Piacente e col., 2002; Lee e col., 2006). Por isso, a constatação do elevado uso da espécie pelos sujeitos em estudo e a evidência da ausência de estudos de validação do uso de Turnera ulmifolia em HIV/AIDS merece atenção. Devem ser considerados os riscos de interferência no tratamento de referência.

Plectranthus barbatus (boldo), de uso referido no presente trabalho, devido à sua ação anticolinérgica, pode aumentar os níveis de efavirenz, induzindo estimulação do sistema nervoso central, provocando efeitos colaterais neuropsiquiátricos (Fetrow e Ávila, 2000).

Matricaria chamomilla (camomila) e Mentha spp. (hortelã), espécies vegetais com indicação de emprego popular nos entrevistados com HIV/AIDS neste estudo, também contêm substâncias que podem inibir as enzimas do citocromo $\mathrm{P} 450$, interferindo na biodisponibilidade e toxicidade dos medicamentos antirretrovirais (Foster e col., 2003.

O risco real de interação das espécies vegetais mais frequentemente referidas pela população em estudo com os antirretrovirais revela a importância de o médico estar atento a tal associação. Portanto, é fundamental a conduta médica orientada no sentido de resgatar as experiências dos pacientes com as plantas, incluindo as espécies empregadas, duração, frequência de uso e efeitos percebidos (Littlewood e Vanable 2008).

Na prática, constata-se que muitos profissionais desconhecem questões sobre o uso de plantas com finalidade terapêutica. Em pesquisa realizada no Estado de Goiás - Brasil, Dutra (2009), para verificar o conhecimento sobre o uso de plantas e fitoterápicos e com base nas determinações normativas vigentes, evidencia este fato, revelando que os médicos foram os profissionais menos conhecedores (17\%) e mais resistentes às práticas alternativas.

Embora o presente estudo não tenha explorado diretamente esta questão na perspectiva do profissional, constatou-se que, ao serem informados pelos pacientes sobre o uso de plantas, 37,93\% dos médicos não expressaram sua opinião: somente 1(um) paciente entrevistado foi orientado a interromper o tratamento com plantas. Isto evidencia que esses profissionais desconhecem e não valorizam as práticas complementares e/ou alternativas, especialmente os efeitos prejudiciais do uso de planta e antirretrovirais simultaneamente.

Não emitir opinião sobre a utilização de plantas com finalidade medicinal e a falta de diálogo com 
o paciente sobre o tema pode ser explicado pela própria formação médica, fortemente influenciada por modelos biomédicos, desconhecendo práticas outras que não as relacionadas a esta perspectiva. (Maliska e Padilha, 2007).

Neste estudo, evidenciou-se que a maioria dos indivíduos (73,77\%) não informou ao médico sobre a utilização de plantas. Este elevado percentual também foi observado nos estudos de Sales e colegas (2008), em que $77 \%$ dos indivíduos com HIV/AIDS não informaram ao médico sobre a utilização de plantas. Littlewood e Vanable (2008) alertam que a utilização de medicina complementar e/ou alternativa, incluindo as plantas, bem como a omissão deste uso, pode derivar da falta de confiança dos pacientes no sistema de saúde, no médico e nos demais profissionais.

Constatou-se, ainda, que a indicação para o uso de plantas partiu principalmente de amigos, família ou conta própria $(56,25 \%)$, comprovando que apenas um médico indicou o uso de plantas no tratamento de HIV/AIDS.

Resultados semelhantes também foram obtidos por Sales e colegas (2008), o qual constatou que $56,6 \%$ dos pacientes entrevistados justificaram o uso de plantas devido a hábitos tradicionais familiares, comprovando que em $83,6 \%$ dos casos a recomendação para utilização de plantas com finalidade medicinal partiu de amigos e parentes. Este autor identificou, ainda, que $4,5 \%$ dos pacientes foram induzidos a essa prática por programas de televisão, anúncios em outdoor, rádio, revistas e jornais, enquanto somente $3 \%$ receberam indicação de uso de plantas por médicos.

Neste estudo foi evidenciado, com significância estatística, que as mulheres utilizaram mais plantas com finalidade medicinal no tratamento do HIV/ AIDS que os homens. Pesquisa semelhante, desenvolvida por Hsiao e colegas (2003), não evidenciou diferença estatisticamente significante entre os sexos para uso de práticas complementares e/ou alternativas.

A predominância de uso de plantas por pessoas do sexo feminino é frequente. Segundo Medeiros e Cabral (2001) e Almassy Júnior (2004), esse fenômeno é decorrente do papel familiar das mulheres, ou seja, estas se responsabilizam mais que os homens por questões relacionadas à saúde e à doença.

Em sociedades tribais, a arte de curar os doentes utilizando o conhecimento popular sobre o poder curativo de plantas, era realizada por mulheres, cujo conhecimento era adquirido no seio familiar (Alvim e col., 2004). Dutra (2009) acrescenta que a prática do cuidado vivida e transmitida de mulher para mulher persiste até os dias de hoje.

Em relação ao uso de plantas e à orientação sexual, esta pesquisa constatou que o uso de plantas com finalidade medicinal nos pacientes com HIV/AIDS entrevistados foi associado à orientação sexual, evidenciando que os entrevistados que realizavam prática sexual do tipo homossexual foram mais propensos ao uso de plantas como recurso terapêutico em relação aos que realizavam prática do tipo heterossexual. Estudos têm evidenciado associação entre prática do tipo homossexual e uso de medicina complementar e/ou alternativa (Hsiao e col., 2003; Littlewood e Vanable, 2008).

Algumas pesquisas têm constatado associação do uso de plantas com outros fatores, tais como: uso de inibidores de protease, tempo de diagnóstico da infecção, ser morador de zona rural e não ter religião (Chang e col., 2003; Colebunders e col., 2003; Peltzer e col., 2008). Na amostra estudada, o uso de plantas não foi associado a nenhum desses fatores, mostrando que, neste serviço, tal prática deve ser explicada por outros fatores que não estes descritos na literatura.

$\mathrm{Na}$ busca de novas alternativas terapêuticas no combate ao HIV, estudos têm sido desenvolvidos avaliando a capacidade antiviral de substâncias ativas presentes em plantas empregadas na prática popular como medicinais (Groweiss e col., 2000).

Em pesquisa desenvolvida no Brasil, Braz-Filho (2010) demonstra a capacidade antiviral de cumarinas, flavonóides e crisinas isolados das flores de Chysanthbermum morifolium (família Compositae). Vale ressaltar que tais pesquisas são preliminares, não havendo, até o momento, estudos de validação terapêutica de espécies vegetais no combate ao HIV. Portanto a terapêutica recomendada é o antirretroviral.

Este estudo apresenta limitação do delineamento transversal, que não permite investigação tipo causa-efeito entre HIV/AIDS e plantas, bem como o 
fato da amostra ser de ambulatório de referência, com população aparentemente homogênea. Assim, os resultados não podem ser generalizados para população geral de pessoas vivendo com HIV/AIDS na área de estudo.

Considera-se, no entanto, que este estudo é relevante para o conhecimento do uso de plantas por pacientes com sorologia positiva para HIV no ambulatório em questão, em tratamento com antirretrovirais, pois pode servir como parâmetro na tomada de decisões, visto revelar o risco real do uso de plantas sem segurança e eficácia terapêutica. Além disso, demonstra a necessidade de capacitação dos profissionais em saúde quanto ao uso racional de plantas com finalidade medicinal e, ainda, como critério de seleção de espécies vegetais para desenvolvimento de estudos de validação.

\section{Referências}

\begin{abstract}
ALMASSY JÚNIOR, A. A. Análise
das características etnobotânicas e etnofarmacológicas de plantas medicinais na comunidade de Lavras Novas, Ouro Preto-MG. 2004. 132 p. Tese (Doutorado em Fitotecnia) Universidade Federal de Viçosa, Viçosa.
\end{abstract}

ALVIM, N. A. T. et al. Tecnologias na enfermagem: o resgate das práticas naturais no cuidado em casa, na escola e no trabalho. In: FIGUEREDO, N. M. A. (Org.). Tecnologias e técnicas em saúde: como e por que utilizá-las no cuidado de enfermagem. São Paulo: Difusão Editora, 2004. v. 1, p. 338-55.

AMARAL, F. M. M.; COUTINHO, D. F.; MESQUITA, R. K. K. Riscos na utilização de plantas para uso medicinal comercializadas em mercados de São Luís/Maranhão. Revista de Ciências da Saúde, São Luís, v. 3, n. 1, p. 37-42, 2001.

AMARAL, F. M. M. Potencial giardicida de espécies vegetais: aspectos da etnofarmacologia e bioprospecção. 2007, 346 p. Tese (Doutorado em Produtos Naturais e Sintéticos Bioativos) Universidade Federal da Paraíba, João Pessoa.
ANTONIO, M. A.; SOUZA BRITO, A. R. M. Oral antiinflammatory and anti-ulcerogenic activities of a hydroalcoholic extract and partitioned fractions of Turnera ulmifolia (Turneracea). Journal of Ethnopharmacology, Lausanne, v. 61, n. 3, p. 21528, 1998.

ARANTES, V. P. Estudo da atividade antimicobacteriana de extratos vegetais do cerrado brasileiro. 2005. 75 f. Dissertação (Mestrado em Análises Clínicas) - Programa de Pós-Graduação em Análises Clínicas, Universidade Estadual Paulista, Araraquara, SP.

BARBOSA, D. A.; SILVA, K. N.; AGRA, M. F. Estudo farmacobotânico comparativo de folhas de Turnera chamaedrifolia Cambess. e Turnera subulata Sm. (Turneraceae). Revista Brasileira de Farmacognosia, João Pessoa, v. 17, n. 3, p. 396-413, 2007.

BARROS, A. J.; HIRAKATA, V. N. Alternatives for logistic regression in cross-sectional studies: an empirical comparison of models that directly estimate the prevalence ratio. BMC Medical Research Methodology, London, v. 3, n. 21, p. 21, 2003.

BRASIL. Decreto $n^{\circ}$ 5.813, de 22 de junho de 2006. Aprova a Política Nacional de Plantas Medicinais e Fitoterápicos e dá outras providências. Diário Oficial da União, Brasília, DF, Seção1, 23 de junho de 2006, p. 2.

BRAZ-FILHO, R. Contribuição da fitoquímica para o desenvolvimento de um país emergente. Química Nova, São Paulo, v. 33, n. 1, p. 229-239, 2010.

CHANG, B. L.; VAN SERVELLEN, G.; LOMBARDI, E. Factors associated with complementary therapy use in people living with HIV/AIDS receiving antirretroviral therapy. Journal of Alternative and Complementary Medicine, London, v. 9, n. 5, p. 695-710, 2003.

CHINSEMBU, K. C. Model and experiences of initiating collaboration with traditional healers in validation of ethnomedicines for HIV/ AIDS in Namibia. Journal of Ethnobiology and Ethnomedicine, London, v. 5, n. 1, p. 30, 2009. 
COLEBUNDERS, R. et al. The use of

complementary and alternative medicine by persons with HIV infection in Europe.

International Journal of STD \& AIDS, London, v. 14, n. 10, p. 672-4, 2003.

COUTINHO, D. F.; TRAVASSOS, L. M. A.;

AMARAL, F. M. M. Estudo etnodirigido de plantas medicinais no Estado do Maranhão - Brasil. Visão Acadêmica, Curitiba, v. 3, n. 1, p. 7-12, 2002.

DUTRA, M. G. Plantas medicinais, fitoterápicos e saúde pública: um diagnóstico situacional em Anápolis, Goiás. Anápolis: Centro Universitário de Anápolis - UniEvangélica, 2009.

ELDIN, S.; DUNFORD, A. Fitoterapia na atenção primária à saúde. São Paulo: Manole, 2001.

FEITOSA, A. C.; TROVÃO, J. R. Atlas escolar do Maranhão: espaço geo-histórico e cultural. João Pessoa: Grafset, 2006.

FETROW, C. W.; ÁVILA, J. R. Manual de medicina alternativa para o profissional. Rio de Janeiro: Guanabara Koogan, 2000.

FOSTER, B. C. et al. In vitro inhibition of human cytochrome P45o- mediated metabolismo of marker substrates by natural products. Phytomedicine, Stuttgard, v. 10, n. 1, p. 334-42, 2003.

GROWEISS, A.; CARDELLINA, J. H., BOYD, M. R. HIV-Inhibitory prenylated xanthones and flavones from tinctoria. Journal of Natural Products, Cincinnati, US, v. 63, p. 1537-9, 2000.

HSIAO, A. F. et al. S. Complementary and alternative medicine use and substitution for conventional therapy by HIV-infected patients. Journal of Acquired Immune Deficiency Syndromes, Hagerstown, v. 33, n. 2, p. 157-65, 2003.

LEE, L. S., ANDRADE, A. S., FLEXNER, C. Interations between natural health products an antirretroviral drugs: pharmacokinetic and pharmacodynamic effects. Clinical Infectious Diseases, Chicago, v. 43, n. 8, p. 1052-9, 2006.
LITTLEWOOD, R. A.; VANABLE, P. A.

Complementary and alternative medicine use among HIV-positive people: research synthesis and implications for HIV care. AIDS Care, London, v. 20, n. 8, p. 1002-18, 2008.

LIU, J. P.; MANHEIMER, E.; YANG, M. Herbal medicines for treating HIV infection and AIDS. Cochrane Database of Systematic Reviews, Oxford, v. 3, 2005.

MACEDO, A. F.; OSHIIWA, M.; GUARIDO, C. F. Ocorrência do uso de plantas medicinais por moradores de um bairro do município de MaríliaSP. Revista de Ciências Farmacêuticas Básica e Aplicada, Araraquara, v. 28, n. 1, p. 123-8, 2007.

MALISKA, I. C. A.; PADILHA, M. I. C. D. S. Aids: a experiência da doença e a construção do itinerário terapêutico. Revista Eletrônica de Enfermagem, Goiânia, v. 9, n. 3, p. 687-99, 2007.

MATOS, F. J. A. Plantas medicinais: guia de seleção e emprego de plantas usadas em fitoterapia no Nordeste do Brasil. 2. ed. Fortaleza: Imprensa Universitária-UFC, 2000. 344 p.

MEDEIROS, L. C. M.; CABRAL, I. E. O cuidar com plantas medicinais: uma modalidade de atenção à criança pelas mães e enfermeira-educadora. Revista Latino-Americana de Enfermagem, Ribeirão Preto, v.9, n. 1, p.18-26, 2001.

MEINERS, C. M.; AZEVEDO, M. Patentes

farmacêuticas e saúde pública: desafios à política brasileira de acesso ao tratamento antirretroviral. Cadernos de Saúde Pública, Rio de Janeiro, v. 24, p. 1467-78, 2008.

MINISTÉRIO DA SAÚDE. Agência Nacional de Vigilância Sanitária. Portaria nº 971, de o3 de maio de 2006. Aprova a Política Nacional de Práticas Integrativas e Complementares (PNPIC) no Sistema Único de Saúde. Diário Oficial da União, Brasília, DF, o4 maio 2006, p. 20.

MONTELES, R.; PINHEIRO, C. U. B. Plantas medicinais em um quilombo maranhense: uma perspectiva etnobotânica. Revista de Biologia e Ciências da Terra, Campina Grande, v. 7, n. 2, p. 38-48, 2007. 
MOURA, E. G. Agroambientes de transição entre o trópico úmido e o semiárido do Brasil. São Luís: UEMA, 2004. 321p.

NASCIMENTO, M. A. et al. Turnera ulmifolia L. (Turneraceae): preliminary study of its antioxidant activity. Bioresource Technology, Barking, v. 97, n. 12, p. 1387-91, 2006.

PASSOS, A. M. et al. Potenciais interferências nos resultados de exames laboratoriais causadas pelo uso de plantas medicinais por pacientes HIV positivos e/ou AIDS. Latin American Journal of Pharmacy, Buenos Aires, v. 28, n. 1, p. 196-202, 2009.

PAULA, P. A. B. et al. Política de medicamentos: da universalidade de direitos aos limites da operacionalidade. Physis, Rio de Janeiro, v. 19, p. 1111-25, 2009.
PELTZER, K. et al. Use of traditional complementary and alternative medicine for HIV patients in KwaZulu-Natal, South Africa. BMC Public Health, London, v. 8, n. 255, p. 78-9, 2008. PIACENTE, S. et al. Flavonoids and arbutin from Turnera diffusa. $Z$ Naturforsch C, Tübingen, v. 57, n. 11-12, p. 983-985, 2002.

RÊGO, T. J. A. S. Fitogeografia das plantas medicinais do Maranhão. 2. ed. São Luís: Edufma, 1995.

SALES, P. M. et al. The use of herbal medicine by AIDS patients from university hospital of the Brasília, Brazil. Boletín Lationoamericano y del Caribe de Plantas Medicinales y Aromáticas, Santiago, v.7, n. 4, p. 208-17, 2008.

YUNES, R. A.; PEDROSA, R. C.; CECHINEL FILHO, V. Fármacos e fitoterápicos: a necessidade do desenvolvimento da indústria de fitoterápicos e fitofármacos no Brasil. Química Nova, São Paulo, v. 24, n. 1, 2001. 